\title{
Biform Analysis of Inventory Competition
}

\author{
Harborne W. Stuart Jr. \\ Columbia Business School, Columbia University, New York, New York 10027, \\ hws7@columbia.edu
}

\begin{abstract}
$\mathrm{T}_{\mathrm{f}}^{\mathrm{h}}$ his paper provides a model of the competitive newsvendor problem in which there is price competition following the inventory decisions. Using the biform game formalism of Brandenburger and Stuart (2004), the price competition is modeled by considering the core of the induced cooperative game. Such an analysis allows price competition to be modeled without a priori assumptions about price-setting power or pricing procedures. The paper shows that with no uncertainty, the inventory decision is equivalent to the capacity decision in Cournot competition. With uncertainty, the analysis again reduces to Cournot competition if the demand uncertainty is characterized by an appropriately constructed, expected demand curve. The results highlight the critical role of the fixed-price assumption in newsvendor models.
\end{abstract}

Key words: biform game; cooperative game; capacity competition; Cournot

History: Received: July 11, 2003; accepted: April 8, 2005. This paper was with the author 9 months for

2 revisions.

\section{Introduction}

The newsvendor model provides a standard approach to analyzing competitive inventory decisions under uncertainty. In such a model, firms precommit to inventory levels before a demand uncertainty is realized. When the demand is realized, the goods are sold at a price that is usually assumed to be fixed. In this paper, the fixed-price assumption is replaced with price competition - the price of the goods is determined by competition between the firms and the buyers.

To model the price competition, this paper uses the biform game formalism of Brandenburger and Stuart (2004). A biform game is a hybrid noncooperative/cooperative game model designed for modeling business interactions. In the application to the newsvendor problem, the choice of inventory level is an explicit move, just as it would be in a noncooperative game. Price competition is then modeled by the core of a cooperative game. By using cooperative game theory to model price competition, the exact procedures of the pricing game do not need to be specified. Examples of specified pricing procedures are simultaneous take-it-or-leave-it offers by firms, sequential take-it-or-leave-it offers by firms, sealed-bid auctions, etc. With any of these procedural models, the results usually depend upon the choice of procedure. By contrast, in the core of a cooperative game, a competitive outcome is obtained without making any procedural assumptions. In fact, the core can be said to model unrestricted competition: "unrestricted," because there are no restrictions on the transactions between players, and "competitive," because in any core outcome there can be no opportunity for a group of players to do a better deal on their own. ${ }^{1}$

This paper investigates the impact of unrestricted competition in a newsvendor context. The results suggest two conclusions. First, fixed retail prices are a defining characteristic of a newsvendor analysis. Although this insight is implicit in many newsvendor analyses, it becomes self-evident in a biform analysis. Second, market-clearing prices are not necessarily implied by unrestricted competition. Although they can arise with unrestricted competition, there is no guarantee that they will. This second conclusion has two practical implications. The first is that care must be taken when modeling price competition

\footnotetext{
${ }^{1}$ This use of cooperative game has a long history, starting with von Neumann and Morgenstern (1944), if not Edgeworth (1881). This use in the "modern" era starts with Shapley and Shubik; see, for example, Shapley and Shubik $(1969,1972)$. More recently, Brandenburger and Stuart (1996) argue for the use of cooperative game theory in modeling business contexts.
} 
with market-clearing prices. The domain in which a market-clearing price can be justified by unrestricted competition is limited. The second implication is that in order to obtain a sharp prediction, a model of inventory competition may benefit from a specified pricing procedure; unrestricted competition may not be the best modeling choice. For if unrestricted competition does not lead to a clear outcome, such as a market-clearing price, the inventory decision can be difficult to formulate. A model with a specified pricing procedure might then provide more insight. In such cases, a model without pricing procedures, namely a biform model, can be used to motivate, perhaps ironically, a model with pricing procedures.

The main result of this paper is that the newsvendor model with price competition reduces to the Cournot duopoly (or oligopoly) problem. By characterizing the demand uncertainty with an appropriately constructed, expected-demand curve, inventory competition takes the form of Cournot capacity competition. This result differs from standard newsvendor results in two significant ways. First, virtually all newsvendor models capture an overage versus underage tension. There is a cost associated with having too many units when demand is lower than desired, and there is a cost associated with having too few units when demand is higher than desired. With unrestricted competition, this tension disappears. In its place, the quantity decision takes the form of a more traditional economic trade-off: fewer units at a higher price versus more units at a lower price. Because the overage/underage tension is such a key element of a newsvendor analysis, this suggests that fixed retail prices are an essential, if not defining, characteristic of newsvendor-style analyses.

This conclusion is further supported by the second significant difference that arises in a biform analysis of the newsvendor problem. When prices are fixed, a newsvendor model must specify a set of rules for how buyers are matched with firms. Different modeling objectives have led to at least four types of approaches. In the first type, each firm is assumed to face a random demand. If there is excess demand, the unsatisfied buyers try to buy from other firms based on fixed proportions, and if an unsatisfied buyer is again unsuccessful in the second attempt, the buyer does not transact. Examples of this approach include Parlar (1988), Karjalainen (1992), and Netessine and Rudi (2003). In the second type of approach, rather than viewing aggregate demand as the sum of individual firm demands, Lippman and McCardle (1997) start with aggregate demand and then allocate it by both random and deterministic rules. In the reallocation of excess demand, they allow for different rules as well, including symmetric reallocations and "herding" reallocations. In the third type, Mahajan and van Ryzin (2001) replace specific allocation and reallocation rules with a complete model of consumer choice. For each buyer, there is a preference over each firm, and for each state of nature, there is an order in which the buyers get to purchase (or not). Finally, buyers have been matched to firms based on the firms' stocking quantities. Motivated by situations in which more shelf space leads to greater sales, Wang and Gerchak (2001) allocate the aggregate demand based on displayed inventories. More broadly, Deneckere and Peck (1995) and Netessine and Zhang (2003) model each firm's effective demand as a function of the stocking decisions of every other firm. Deneckere and Peck (1995) consider a specific function based on service levels (and prices), and Netessine and Zhang (2003) consider general functions.

In a biform analysis, the question of how to model the matching of buyers with firms does not have to be addressed. Since the competition is unrestricted, the goods must end up in the hands of the buyers who value them the most. To emphasize the extent to which a core analysis puts minimal restrictions on price competition, it is worth noting that other forms of price competition may not solve the matching problem. For example, in a model with deterministic demand, Kreps and Scheinkman (1983) model firms which first make capacity choices, and then compete with Bertrand pricing. They derive an equilibrium result in which firms first choose Cournot quantities and then set a price equal to the corresponding, market-clearing price. But to derive this result, they have to specify what would happen if firms were to price above the market-clearing price. They require that in such cases the goods be sold efficiently, namely that the goods be sold to the buyers who value them the most. However, Davidson and Deneckere (1986) show that with other allocation 
rules, the Cournot result will not necessarily obtain. ${ }^{2}$ In the biform analysis of capacity choice, a matching rule is not required. Instead, matchings are a consequence of the model of competition.

When the core of a game is nonempty, a core outcome can be described by prices paid and received by the players. In general, these prices can be different for different players, but in the model of this paper, the core analysis yields a tighter result: A market-clearing price will always emerge. Consequently, a biform analysis provides a foundation for models which assume the existence of a marketclearing price. For example, Van Mieghem and Dada (1999), while investigating postponement strategies under uncertainty, consider a model ("Price Postponement with Clearance") in which firms must first commit to capacity choices, but then a market-clearing price is assumed to emerge. Based on the current paper's results, unrestricted competition can be used as a foundation for their market-clearing assumption, and their result can be viewed as a continuous application of the main result of the current paper. Unfortunately, unrestricted competition does not always guarantee market-clearing prices. Two situations are particularly relevant. If demand is not unitary, in other words if some buyers want more than one unit, then a market-clearing price will not necessarily emerge. And, even when demand is unitary, if buyers view the products of the firms differently, either due to transportation costs or actual differences, then unrestricted competition will tend to generate outcomes with buyer-specific prices rather than market-clearing prices. This leads to the second conclusion, namely that modeling price competition with the assumption of market-clearing prices can be problematic. An additional assumption of unitary demand supports the Van Mieghem and Dada (1999) result, but other results may be harder to support. For instance, Chod and Rudi (2003) extend the Van Mieghem and Dada model to account for resource flexibility in a multiproduct context. Because their model allows for substitution (and complementarity), a core analysis is likely to generate more than just market-clearing prices. Thus, their assumption of

\footnotetext{
${ }^{2}$ The literature on Cournot and related oligopoly models is extensive. For an introduction, see Tirole (1988) and Vives (1999).
}

market-clearing prices rests on a model of price competition different from the unrestricted competition of a core analysis.

This paper uses cooperative game theory to model unrestricted price competition, but other papers have used cooperative game theory differently in their analysis of competitive inventory problems. For example, Wang and Parlar (1994), in a model of inventory competition with fixed prices, use cooperative game theory in one of its original uses: They start with a noncooperative game, then suppose that the players can cooperate on strategy choices. In another use of cooperative theory, Müller et al. (2002) use the core to show that there is always a cost allocation scheme such that newsvendors will prefer to pool their inventory. More recently, Anupindi et al. (2001) use a hybrid noncooperative/cooperative model similar to a biform game. In their interpretation of their model, they use cooperative game theory to characterize possible opportunities for cooperation, similar to Müller et al. (2002). By contrast, the use of cooperative game theory in a biform game has very little to do with cooperation per se. It is not derived from supposing that players can cooperate on their strategy choices. Rather, the cooperative game is treated as a primitive, providing an abstract model of a competitive environment. (See Aumann 1985 for a further discussion of this use of cooperative game theory.) To emphasize this point, $\$ 2$ demonstrates how the core models unrestricted competition.

In $\S 3$, the biform formalism is introduced, and the model is specified. Section 4 contains the main result, namely that under unrestricted price competition, competitive inventory choices reduce to Cournot competition. Section 5 provides examples demonstrating why market-clearing prices may not emerge as often as one might suspect. Additional examples show that if the discreteness of demand creates indeterminacy in the outcome, preemptive capacity decisions can be optimal. The paper ends with concluding remarks in $\S 6$.

\section{Core as a Model of Price Competition}

A transferable utility (TU) cooperative game $(N ; v)$ consists of a set of players, $N$, and a characteristic function, $v: 2^{N} \rightarrow \Re$. The characteristic function specifies 
the economic value that can be created by any subset of players, i.e., for any $S \subseteq N, v(S)$ is the maximum economic value that the players in $S$ can create among themselves. An outcome of a TU cooperative game is described by an allocation $x \in \mathfrak{R}^{|N|}$, where component $x_{i}$ denotes the value received by player $i$. For notational ease, let $x(S)=\sum_{i \in S} x_{i}$. The core of a TU cooperative game $(N ; v)$ is the set of allocations satisfying $x(N)=v(N)$ and, for all $S \subseteq N, x(S) \geq v(S)$.

The following elementary example demonstrates the use of the core to model price competition. It also previews how with unitary demand, a marketclearing price must emerge.

EXAMPLE 1. Suppose there are three buyers, each of whom has a willingness-to-pay for just one unit of product. Buyer 1 has a willingness-to-pay of 8 , denoted by $w_{1}$, Buyer 2 has a willingness-to-pay of 6 , denoted by $w_{2}$, and Buyer 3 has a willingness-to-pay of 4 , denoted by $w_{3}$. There are two firms, say $A$ and $B$, and suppose each has installed one unit of capacity at a cost of zero, and each has a constant marginal production cost of 2 , denoted by $c$. The player set $N$ is then $\{A, B, 1,2,3\}$, and $v(N)=10$, namely $(8-2)+$ $(6-2)$. Figure 1 depicts the situation.

Because the value that can be created is 10 , and because $v(\{A, B, 1,2\})$ is also equal to 10 , Buyer 3 is superfluous to the value creation. This suggests that $x_{3}$ should be equal to 0 , and this is the case: the core conditions $x(N)=v(N)$ and $x(N \backslash\{3\}) \geq$ $v(N \backslash\{3\})=10$ imply that $x_{3}=0$. Nonetheless, Buyer 3 will still have an effect on the outcome by providing a source of competition. This will be shown below.

To show how a market-clearing price must emerge, first consider an allocation consistent with Buyers 1

\section{Figure 1 Supply and Demand in Example 1}

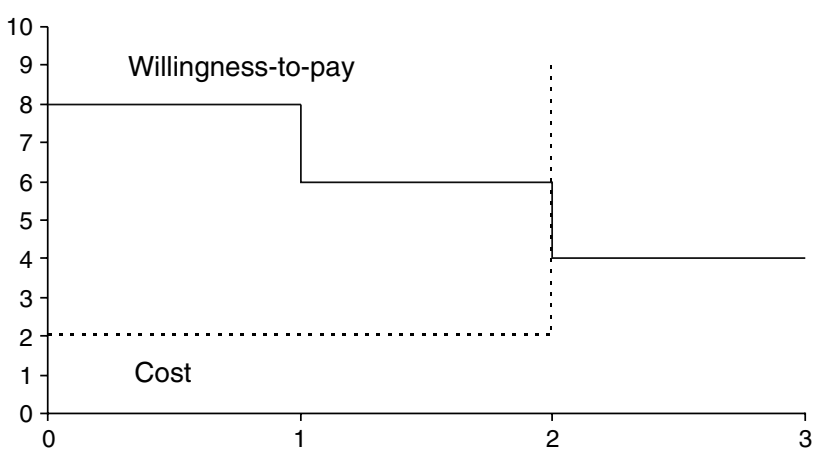

and 2 paying different prices:

$$
\begin{aligned}
& x_{A}=\left(p_{1}-c\right)=\left(p_{1}-2\right) \\
& x_{B}=\left(p_{2}-c\right)=\left(p_{2}-2\right) \\
& x_{1}=\left(w_{1}-p_{1}\right)=\left(8-p_{1}\right) \\
& x_{2}=\left(w_{2}-p_{2}\right)=\left(6-p_{2}\right) \\
& x_{3}=0 .
\end{aligned}
$$

In this allocation, Buyer 1 can be thought of as buying from Firm $\mathrm{A}$ at price $p_{1}$, and Buyer 2 as buying from Firm $B$ at price $p_{2}$. Now suppose that $p_{1}>p_{2}$. The core not only requires $x(\{A, 1\}) \geq v(\{A, 1\})$ and $x(\{B, 2\}) \geq v(\{B, 2\})$, it also requires, among other conditions, that $x(\{A, 2\}) \geq v(\{A, 2\})$ and $x(\{B, 1\}) \geq$ $v(\{B, 1\})$. Although the first two conditions are met, one of the second two is not met. In words, this allocation is not stable because Firm B and Buyer 1 can do a better deal on their own:

$$
\begin{aligned}
x_{B}+x_{1} & =\left(p_{2}-c\right)+\left(w_{1}-p_{1}\right) \\
& =\left(w_{1}-c\right)+\left(p_{2}-p_{1}\right) \\
& <\left(w_{1}-c\right)=v(\{B, 1\}) .
\end{aligned}
$$

This is an example of why the core can be thought of as modeling competition. A possible allocation has to "compete" with every possible deal. With similar reasoning, it follows that $p_{1}$ cannot be less than $p_{2}$, and so the allocations must be consistent with a uniform price, namely $\left(w_{1}-x_{1}\right)=\left(w_{2}-x_{2}\right)=p$, where $p$ is a constant.

To show that the emergent uniform price is a market-clearing price, note that the intersection of the "supply" and "demand" curves is described by $p \in[4,6]$. Thus, we need to show that $p \in[4,6]$, namely $p \in\left[w_{3}, w_{2}\right]$. First suppose $p>6$. Because $w_{2}=6$, this would imply that $x_{2}<0$, violating the core condition $x_{2} \geq v(\{2\})=0$. (This particular core condition is called individual rationality.) For the lower bound on the price, the potential competition from the "superfluous" Buyer 3 is now considered. Suppose that $p<4$. Then

$$
\begin{aligned}
x(\{A, B, 1,3\}) & =x(\{A, B\})+x_{1}+x_{3} \\
& =2(p-2)+(8-p)+0 \\
& =p+4 \\
& <8=v(\{A, B, 1,3\}),
\end{aligned}
$$


which is impossible in the core. Thus, $p \in[4,6]$, and the core analysis leads to outcomes that can be interpreted as market-clearing prices. Finally, note that $p \in[4,6]$ implies that the market-clearing price is not unique. A single price will emerge, but due to the discreteness of the demand, there is some indeterminacy about what the price will be.

The arguments in this example are not a complete proof, and in particular, the above reasoning has to be enhanced when a firm can sell more than one unit. However, the example previews the formal results, especially the fact that, with unitary demand, the prices are the same as the prices that arise under Cournot competition. To emphasize this point, it will be useful to denote the number of units that will be sold by $q$. In situations with industry undersupply, $p$ will be between $w_{q+1}$ and $w_{q}$, as above. With industry oversupply, $p$ will be shown to be equal to the marginal cost, namely $c$.

\section{Formalism and Model}

A. Biform Formalism. To define a biform game, consider a set of players $N$, indexed by $i=1, \ldots, n$, and for each player $i$, a finite set $A_{i}$ of strategies. Let $A=A_{1} \times \cdots \times A_{n}$, with typical element $a$, and let $V$ be a map from $A$ to the set of maps from $2^{N}$ to $\Re$, where $V(a)(\varnothing)=0$ for every $a \in A$. Note that for a given $a \in A, V(a): 2^{N} \rightarrow \Re$, so that $(N ; V(a))$ is a TU cooperative game. For each player $i$, let $\alpha_{i}$ be a number in $[0,1]$. An n-person biform game is then a collection $\left(A_{1}, \ldots, A_{n} ; V ; \alpha_{1}, \ldots, \alpha_{n}\right)$.

In the two models below, the strategy spaces $A_{i}$ will represent the possible capacity choices for each firm. Based on each firm's choice, the resulting profile of choices, $a \in A$, will define a TU cooperative game $V(a): 2^{N} \rightarrow \Re$. Competition is then modeled by the core of the cooperative game. As noted in the introduction, this combined use of a TU cooperative game with a core analysis is a standard method for modeling price competition without a priori assumptions of price-setting power or procedures. If a core analysis yields a range of outcomes, rather than a unique outcome, then the consequence of a player's action typically will be an interval of possible payoffs, rather than a unique payoff. In such cases, it is necessary to describe each player's preferences over intervals. In a biform game, these preferences are represented by the numbers $\alpha_{i}$ (i's confidence index) for each player $i$. Confidence indices will be discussed in further detail in $\S 5.2$.

Before introducing demand uncertainty, it is useful to first consider a model with known demand. This is done in the next subsection. As will be seen, the uncertainty can be formulated in such a way that extending the model to cover uncertainty is straightforward. This is shown in the following subsection.

B. Oligopoly Model. Let $N=F \cup T$, where $F \cap T=$ $\varnothing,|F| \geq 2$, and $T=\{1, \ldots, b\}$, where $b>1$. The set $F$ is the set of firms, and the set $T$ is the set of buyers. Let $w_{j}(j=1, \ldots, b)$ be numbers satisfying $w_{1} \geq \cdots \geq$ $w_{b} \geq 0$, where each $w_{j}$ is buyer $j$ 's willingness-to-pay for a unit of product from any of the firms. The plot of the $w_{j}$ versus $j$ describes a discrete demand curve. For each firm, namely each $i \in F$, denote its strategy set by $A_{i}=\{0,1,2, \ldots\}$. A firm's strategic choice, namely an $a_{i} \in A_{i}$, is its choice of capacity. The buyers do not have a strategic choice. ${ }^{3}$

Let $k \geq 0$ be the constant unit cost of capacity, and let $c \geq 0$ denote the constant marginal cost of production. In the inventory-competition interpretation of this model, each $a_{i} \in A_{i}$ is a choice of inventory quantity, each unit of inventory costs $k+c$, and each unit has a salvage value of $c$. Thus, the cost associated with an unsold unit would be $k$, and the cost associated with a sold unit would be $(c+k)$, just as in the capacity interpretation.

To simplify the specification of the characteristic functions $V(a)$, consider an $a \in A$. Then, for any $S \subseteq N$, let $a_{S}=\sum_{i \in S \cap F} a_{i}$, let

$$
w(S)=\left\{j \in S \cap T: w_{j} \geq c\right\},
$$

let $d_{S}=|w(S)|$, and let

$$
R(S)=\max \left\{r: \sum_{j=1}^{r} \chi_{S}(j) \leq \min \left\{a_{S}, d_{S}\right\}\right\},
$$

where the function $\chi_{S}$ is the characteristic function of $S$. $\left(\chi_{S}(j)=0\right.$ or 1 according as $j \notin S$ or $j \in S$, where $j=1, \ldots, b$.)

\footnotetext{
${ }^{3}$ To be consistent with the definition of a biform game, each buyer should be given a singleton strategy set. For simplicity, these singleton strategy sets are omitted.
} 
The term $a_{S}$ is the total capacity of the firms in coalition $S$, the set $w(S)$ contains the "viable" buyers in $S$, namely those who could generate a nonnegative gain from trade, and the term $d_{S}$ is the total viable demand in $S$. In particular, note that $a_{N}$ can be thought of as the aggregate supply and $d_{N}$ as the aggregate demand. The term $R(S)$ is used to identify the buyers in $S$ that would transact with the firms, if the game were to consist only of the players in $S$.

Given an $a \in A$, the characteristic function $V(a)$ is defined as follows. For all $S \subseteq N$,

$$
V(a)(S)= \begin{cases}0 & \text { if } S \cap F=\varnothing, \\ -k a_{S} & \text { if } S \cap T=\varnothing, \\ -k a_{S}+\sum_{j=1}^{R(S)} \chi_{S}(j)\left(w_{j}-c\right) & \text { otherwise. }\end{cases}
$$

C. Oligopoly with Uncertainty. To model uncertainty with the biform game formalism, nature is modeled as a player, namely $\varphi$. Consider a player set $N^{\prime}=N \cup\{\varphi\}$, where $N$ is the player set defined above. Also, let the sets $A_{i}$ be defined as above, but let $\Omega$, rather than $A_{\varphi}$, denote the strategy set of nature, namely player $\varphi$. For each $\omega \in \Omega$, let $W_{1}(\omega), W_{2}(\omega), \ldots, W_{b}(\omega)$ be a sequence of nonnegative numbers with $W_{1}(\omega) \geq W_{2}(\omega) \geq \cdots \geq W_{b}(\omega)$. (For a given $\omega$, the plot of the $W_{j}(\omega)$ versus $j$ describes a discrete demand curve.)

Similar to before, consider an $(a, \omega) \in A \times \Omega$. Let

$$
W(\omega)(S)=\left\{j \in S \cap F: W_{j}(\omega) \geq c\right\},
$$

let $d_{S}=|W(\omega)(S)|$, and let

$$
R(S)=\max \left\{r: \sum_{j=1}^{r} \chi_{S}(j) \leq \min \left\{a_{S}, d_{S}\right\}\right\} .
$$

(The value of $d_{S}$, and so $R(S)$ as well, depends on $\omega$, but because the values will be clear from the context, this dependence is suppressed in the notation.)

Given an $(a, \omega) \in A \times \Omega$, the characteristic function $V^{\prime}(a, \omega)$ is defined as follows. For all $S \subseteq N$,

$$
V^{\prime}(a, \omega)(S)= \begin{cases}0 & \text { if } S \cap F=\varnothing, \\ -k a_{S} & \text { if } S \cap T=\varnothing, \\ -k a_{S}+\sum_{j=1}^{R(S)} \chi_{S}(j)\left(W_{j}(\omega)-c\right) & \text { otherwise. }\end{cases}
$$

Additionally, for all $S \subseteq N$,

$$
V^{\prime}(a, \omega)(S \cup\{\varphi\})=V^{\prime}(a, \omega)(S) .
$$

In the interpretation of the model with uncertainty, the firms first simultaneously choose capacity, then nature moves, i.e., the uncertainty is resolved, and then there is price competition, modeled by the core of the game $\left(N^{\prime} ; V^{\prime}(a, \omega)\right)$.

Note that for a given $\omega \in \Omega$, the model reduces to the original oligopoly model. Additionally, note that by Equation (2), the model implicitly covers situations in which the number of buyers is uncertain. For, if at a given $\omega \in \Omega, W_{j}(\omega) \leq c$, then buyer $j$ has no effect on the game. Consequently, buyer $j$ can be considered as not in the game at state $\omega$. Further, the model can be related to a more traditional characterization of demand-curve uncertainty as follows. Suppose that the uncertain demand is taken to be a linear function of price with an uncertain constant, for example,

$$
D(p ; \omega)=a+\varepsilon(\omega)-b p,
$$

where $\varepsilon$ is a random variable. In the model above, this would correspond to

$$
W_{j}(\omega)=\frac{a+\varepsilon(\omega)-j}{b} .
$$

\section{Results}

Consider an $a \in A$. Let $q=\min \left\{a_{N}, d_{N}\right\}$. The following lemma describes what firms receive in the core when more than one firm chooses to install capacity.

Lemma 1. For any $a \in A$ such that $\left|\left\{i: a_{i}>0\right\}\right| \geq 2$, consider the cooperative game $(V(a) ; N)$ of Equation (1). The game has a nonempty core, and each firm i receives

$$
a_{i}[p-(k+c)],
$$

where

(i) $p \in\left[w_{q+1}, w_{q}\right]$ if $a_{N}<d_{N}$ ，

(ii) $p \in\left[c, w_{q}\right]$ if $a_{N}=d_{N}$,

(iii) $p=c$ if $a_{N}>d_{N}$.

This result follows from Theorem II in Kaneko (1976). A proof for this specific model is in the appendix.

Lemma 1 states that when more than one firm chooses to install capacity, the model reduces to 
Cournot competition. If only one firm chooses to install capacity, Theorem III in Kaneko (1976) implies the following monopoly result. ${ }^{4}$

LEMMA 2. In the cooperative game $(V(a) ; N)$ of Equation (1), if there exists a firm $i$ such that $a_{i}=a_{N}$, then the core is nonempty, and firm i receives

(i) between $a_{i}\left[w_{q+1}-(k+c)\right]$ and $\sum_{j=1}^{q}\left[w_{j}-(k+c)\right]$ if $a_{i}<d_{N}$,

(ii) between $-a_{i} k$ and $-a_{i} k+\sum_{j=1}^{q}\left[w_{j}-c\right]$ if $a_{i} \geq d_{N}$. All the other firms receive 0 .

The informal reasoning in the discussion of Example 1 is based on Lemma 1 . With industry undersupply, the price will be between the willingnesses-topay of the just-excluded buyer and the just-included buyer, namely, between $w_{q+1}$ and $w_{q}$. When supply matches demand exactly, there is no just-excluded buyer, and the price is between the marginal cost and the willingness-to-pay of the just-included buyer, namely, $c$ and $w_{q}$. When there is excess supply, the price is equal to the marginal cost. When only one firm installs capacity, Lemma 2 shows that a uniform price does not necessarily emerge. But, with undersupply, i.e., part (i), the value $w_{q+1}$ can be interpreted as a minimum price guaranteed to the firm. With this interpretation, note that the value $a_{i}\left[w_{q+1}-(k+c)\right]=$ $q\left[w_{q+1}-(k+c)\right]$ is similar to the profit achieved by a price-setting monopolist, namely $q\left[w_{q}-(k+c)\right]$.

Before stating the main result, it is useful to restate Lemma 1. Note that parts (i) through (iii) of the lemma can be summarized by

$$
p \in[\underline{p}, \bar{p}]
$$

where

$$
\bar{p}=\max \left\{w_{a_{N}}, c\right\}, \quad \underline{p}=\max \left\{w_{a_{N}+1}, c\right\},
$$

and where $w_{j}$ is defined to be equal to $c$ for $j>b$. With this restatement, it becomes clear that from the decision-making perspective of a firm, a willingnessto-pay less than the marginal cost has the same effect as a willingness-to-pay equal to the marginal cost. Using this insight, we can construct an expected,

\footnotetext{
${ }^{4}$ Brandenburger and Stuart (1992) provide a proof of Lemma 2 for this specific model. Moulin (1995, pp. 86-88) derives results similar to Lemmas 1 and 2 for models without a specified characteristic function.
}

"adjusted" demand curve as follows. For $1 \leq j \leq b$, and for a given probability measure $\mu$ on $\Omega$, let

$$
p_{j}=\sum_{\omega \in \Omega} \mu(\omega) \max \left\{W_{j}(\omega), c\right\} .
$$

To simplify the statement of the propositions, for all $j$ such that $b<j \leq b|F|$, let $p_{j}=c$.

The main result can now be stated as follows.

Proposition 1. For any $a \in A$, consider the cooperative game $\left(V^{\prime}(a, \cdot) ; N^{\prime}\right)$ defined by Equations (2) and (3). Suppose nature is playing a mixed strategy $\mu$. If $\left|\left\{i: a_{i}>0\right\}\right|$ $\geq 2$, the expectation over the upper bounds of firm i's core allocations is

$$
a_{i}\left[p_{a_{N}}-(k+c)\right],
$$

and the expectation over the lower bounds of firm i's core allocations is

$$
a_{i}\left[p_{a_{N}+1}-(k+c)\right] .
$$

Proposition 1 states that the capacity choice under uncertainty reduces to Cournot capacity choice under an (appropriately constructed) demand curve. The main reason this result reduces to a simple characterization is due to Lemma 1 . Because a uniform price must emerge in the core, each firm's profit becomes a linear function of price. The proof of Proposition 1 is almost immediate.

Proof. Consider the minimum amount that a given firm, say $i$, will receive. (The argument for the maximum is similar.) By Lemma 1 , for a given $\omega \in \Omega$ and $a \in A$, firm $i$ receives at least $a_{i}\left[\max \left\{W_{a_{N}+1}(\omega), c\right\}-\right.$ $(k+c)]$. Taking the expectation over all $\omega \in \Omega$, firm $i$ receives at least

$$
\begin{aligned}
\sum_{\omega \in \Omega} \mu & (\omega)\left(a_{i}\left[\max \left\{W_{a_{N}+1}(\omega), c\right\}-(k+c)\right]\right) \\
\quad= & a_{i}\left[\left(\sum_{\omega \in \Omega} \mu(\omega) \max \left\{W_{a_{N}+1}(\omega), c\right\}\right)-(k+c)\right] \\
= & a_{i}\left[p_{a_{N}+1}-(k+c)\right] .
\end{aligned}
$$

Since the competitive newsvendor with price competition reduces to a Cournot problem, a natural question is whether a standalone newsvendor problem with price competition reduces to a standard monopoly problem. The answer is positive in the sense that Lemma 2 has a natural extension for uncertain demand, as shown in Proposition 2. 
Proposition 2. For any $a \in A$, consider the cooperative game $\left(V^{\prime}(a, \cdot) ; N^{\prime}\right)$ defined by Equations (2) and (3). Suppose nature is playing a mixed strategy $\mu$. If there exists a firm $i$ such that $a_{i}=a_{N}$, then the expectation over the upper bounds of firm i's core allocations is

$$
-a_{i} k+\sum_{j=1}^{a_{i}}\left(p_{j}-c\right)
$$

and the expectation over the lower bounds of firm i's core allocations is

$$
-a_{i} k+a_{i}\left(p_{a_{i}+1}-c\right) .
$$

Similar to Lemma 2, if the firm is maximizing its minimum expected profit, then it will act like a pricesetting monopolist and maximize $a_{i}\left[p_{a_{i}+1}-(k+c)\right]$. As before, the proof is almost immediate.

Proof. By Lemma 2, for a given $\omega \in \Omega$ and $a \in A$, firm $i$ receives at least $a_{i}\left[\max \left\{W_{a_{N}+1}(\omega), c\right\}-(k+c)\right]$. Taking the expectation over all $\omega \in \Omega$, firm $i$ receives, in expectation, at least

$$
\begin{aligned}
\sum_{\omega \in \Omega} \mu & (\omega)\left(a_{i}\left[\max \left\{W_{a_{N}+1}(\omega), c\right\}-(k+c)\right]\right) \\
& =a_{i}\left[\left(\sum_{\omega \in \Omega} \mu(\omega) \max \left\{W_{a_{N}+1}(\omega), c\right\}\right)-(k+c)\right] \\
& =a_{i}\left[p_{a_{N}+1}-(k+c)\right] .
\end{aligned}
$$

For the expectation over the upper bounds, first note that for cases in which $a_{i}>d_{N}$,

$$
\sum_{j=1}^{q}\left(W_{j}(\omega)-c\right)=\sum_{j=1}^{a_{i}}\left(\max \left\{W_{j}(\omega), c\right\}-c\right) .
$$

From Lemma 2, for a given $\omega \in \Omega$ and $a \in A$, firm $i$ receives at most $-a_{i} k+\sum_{j=1}^{a_{i}}\left(\max \left\{W_{j}(\omega), c\right\}-c\right)$. Taking the expectation over all $\omega \in \Omega$, firm $i$ receives, in expectation, at most

$$
\begin{aligned}
\sum_{\omega \in \Omega} \mu(\omega)\left(-a_{i} k+\sum_{j=1}^{a_{i}}\left(\max \left\{W_{j}(\omega), c\right\}-c\right)\right) \\
=-a_{i} k+\left(\sum_{j=1}^{a_{i}}\left[\sum_{\omega \in \Omega} \mu(\omega) \max \left\{W_{j}(\omega), c\right\}\right]-c\right) \\
=-a_{i} k+\sum_{j=1}^{a_{i}}\left(p_{j}-c\right) .
\end{aligned}
$$

Proposition 2 shows that a competitive inventory problem is not needed to highlight the role of the fixed-price assumption in a newsvendor model; a single-firm problem would have sufficed. However, with a single firm, unrestricted competition leads to results that range from an outcome consistent with a market-clearing price to an outcome consistent with perfect price discrimination. Consequently, a competitive model provides a more structured environment for comparisons. Moreover, as Lemma 2 and Proposition 2 show, unrestricted competition is not sufficient to justify a market-clearing price in a single-firm problem.

\section{Discussion}

\subsection{Market-Clearing Prices}

When the core yields a market-clearing price, and only a market-clearing price, unrestricted competition provides a foundation for models assuming such prices. As noted in the introduction, the "Price Postponement with Clearance" model of Van Mieghem and Dada (1999) provides an example. In particular, their Equation (35) can be viewed as a continuous application of Proposition 1. Unfortunately, Lemma 1 does not hold when demand is generalized to the nonunitary case. Consider the following example.

ExAmple 2. Suppose there are three buyers, say 1,2 , and 3, each of whom desires three units at a willingness-to-pay of one per unit. There are two firms, say $A$ and $B$, and each has installed four units of capacity at a cost of 0 , and each has a constant marginal production cost of 0 .

In this example, there is excess demand $(9>8)$, and the market-clearing price is 1 , implying that each buyer receives no value. Furthermore, this example is seemingly well behaved: The firms are identical and the buyers are identical. However, even though the outcome corresponding to a market-clearing price, namely $\left(x_{A}, x_{B}, x_{1}, x_{2}, x_{3}\right)=(4,4,0,0,0)$, is in the core, every other point in the core is inconsistent with the market-clearing price. Moreover, many of the points in the core are not consistent with even a uniform price, much less the market-clearing price. For instance, consider the following core allocation: $\left(x_{A}, x_{B}, x_{1}, x_{2}, x_{3}\right)=(2.25,2,1.3,1.25,1.2)$. There is no price $p$ consistent with this core allocation, and all the buyers are receiving value, even though there is excess demand. As this example shows, when 
demand is nonunitary, unrestricted competition does not guarantee market-clearing prices.

Example 2 might suggest that as long as demand is unitary, unrestricted competition will produce a market-clearing price, but this is not the case. Consider the case in which buyers have a different willingness-to-pay for each firm's product. The difference could be due to transportation costs, or more generally, to differences in taste. As with nonunitary demand, the unrestricted competition of the core can lead to nonuniform prices. Consider this next example, in which demand is unitary, but some buyers value the firms' products differently.

ExAmple 3. Suppose there are three buyers, each of whom has a willingness-to-pay for just one unit of product. There are two firms, say $A$ and $B$, and each has installed three units of capacity at a cost of 0 , and each has a constant marginal production cost of 0 . Buyer 1 has a willingness-to-pay of 2 for Firm A's product, and a willingness-to-pay of 0 for Firm B's product. Buyer 2 has a willingness-to-pay of 0 for Firm A's product, and a willingness-to-pay of 2 for Firm B's product. Buyer 3 has a willingness-to-pay of 1 for either firm's product.

In this example, every point in the core has the form $\left(x_{A}, x_{B}, x_{1}, x_{2}, x_{3}\right)=\left(p_{A}, p_{B}, 2-p_{A}, 2-p_{B}, 1\right)$, where $p_{A}$ and $p_{B}$ are between 0 and 2. Because Buyer 3 effectively pays a price of zero, there is only one point in the core consistent with a uniform price, namely $p_{A}=p_{B}=0$.

As these examples show, the assumption of a market-clearing price can often be stronger than expected. Without unitary demand and identical products, a market-clearing price needs more than unrestricted competition for justification. But, when a core analysis does generate only a market-clearing price, the analysis can lead to immediate results, as shown by Proposition 1.

\subsection{Indeterminacy}

In the Cournot result of Proposition 1, there is indeterminacy in the price due to the discreteness of the aggregate demand curve. Usually, this type of discreteness is either eliminated by taking an appropriate limit, or avoided by assuming a continuous demand curve. As both of these approaches may be undesirable with a limited number of buyers, we consider two examples that focus on the indeterminacy generated by the discreteness of the demand. We first note that the core, when nonempty, usually describes a set of points rather than a single point. In a biform game, this implies that the consequence of a player's action will be a range of outcomes, rather than a single payoff. If a player is to evaluate different actions, it must have preferences over different ranges. Appendix B of Brandenburger and Stuart (2004) shows that if a player's preferences satisfy four standard axioms (order, dominance, continuity, and positive affinity), then these preferences can be represented by a number between 0 and 1, namely the player's confidence index, denoted by $\alpha_{i}$ for player $i$. Similar to how a player's utility function allows it to evaluate payoffs under uncertainty, a player's confidence index allows it to evaluate different ranges of outcomes. For example, if player $i$ 's possible core outcomes lie in the interval $[r, s]$, then player $i$ evaluates this range by the weighted average $\alpha_{i} s+\left(1-\alpha_{i}\right) r$.

Example 4 demonstrates that with highly confident players, preemptive equilibria can be possible.

Example 4. Suppose there are three buyers, each with a willingness-to-pay for just one unit. Let $w_{1}=14, w_{2}=10, w_{3}=6$. The two firms, $A$ and $B$, each have a constant marginal production cost of 0 . Tables 1a and $1 \mathrm{~b}$ below list each firm's anticipated payoff for capacity choices of 0 to 3 . In each table, $\alpha_{A}=\alpha_{B}$. Equilibrium outcomes are in bold type.

In this example, when $\alpha_{A}=\alpha_{B}=1$, there are two types of equilibria. There are (asymmetric) Cournot equilibria, namely $(2,1)$ and $(1,2)$, and there are two

\begin{tabular}{ccccc} 
Table 1a & $\alpha_{A}=\alpha_{B}=1$ \\
\hline & 0 & 1 & 2 & 3 \\
\hline 0 & 0,0 & 0,14 & 0,24 & $\mathbf{0 , 3 0}$ \\
1 & 14,0 & 10,10 & $\mathbf{6 , 1 2}$ & 0,0 \\
2 & 24,0 & $\mathbf{1 2 , 6}$ & 0,0 & 0,0 \\
3 & $\mathbf{3 0 , 0}$ & 0,0 & 0,0 & 0,0 \\
\hline
\end{tabular}

\begin{tabular}{ccccc} 
Table 1b & \multicolumn{3}{c}{$\alpha_{A}=\alpha_{B}=0.5$} \\
\hline & 0 & 1 & 2 & 3 \\
\hline 0 & 0,0 & 0,12 & 0,18 & 0,15 \\
1 & 12,0 & $\mathbf{8 , 8}$ & 3,6 & 0,0 \\
2 & 18,0 & 6,3 & 0,0 & 0,0 \\
3 & 15,0 & 0,0 & 0,0 & 0,0 \\
\hline
\end{tabular}


preemptive equilibria, namely $(3,0)$ and $(0,3)$. When $\alpha_{A}=\alpha_{B}<0.75$, the asymmetric Cournot equilibria drop out, and a symmetric Cournot equilibrium $(1,1)$ appears. The preemptive equilibria drop out when $\alpha_{A}=\alpha_{B}<2 / 3$. (For an example of the payoff calculation, consider the equilibrium outcome in Table $1 \mathrm{~b}$. The value of $q$ equals 2 , so $p \in\left[w_{3}, w_{2}\right]=[6,10]$. Thus, $\alpha_{i} 10+\left(1-\alpha_{i}\right) 6=8$. $)$

Example 4 also shows how indeterminacy, more precisely a player's preferences over indeterminacy, can affect a firm's choice of capacity. But this does not always have to be the case. Consider the next example with linear demand.

ExAmple 5. Suppose there are $f$ firms and $b$ buyers, where each buyer has a willingness-to-pay for just one unit, and each firm has constant marginal production cost of 0 . For $j \in\{1, \ldots, b\}$, suppose $w_{j}=$ $K-m j$, where $m$ is a positive integer, $K-m b>0$, and $\operatorname{Kmod}(f+1) m \leq m$. For all $i \in F$, set $a_{i}^{*}$ equal to the integer part of $K /((f+1) m)$. Then $a^{*}$ is an equilibrium, regardless of the value of each firm's confidence index. (The proof is in the appendix.)

\subsection{Other Generalizations}

In the market-clearing discussion above, the two examples address the main problems that arise in generalizing the demand. Because it is usually assumed that a firm's cost does not depend upon whom it sells to, cost generalization creates fewer problems. If production costs exhibit decreasing returns to scale, the marginal cost in parts (ii) and (iii) of Lemma 1 will be the "industry" marginal cost, rather than a constant. If there are increasing returns to scale, characterizing a player's core allocation becomes difficult, but proving nonemptiness of the core can be relatively straightforward: A core allocation is created by assigning to each firm an average unit cost and an appropriate revenue. ${ }^{5}$ However, if there are differentiated products as well as increasing returns to scale, the core may be empty. ${ }^{6}$

Another form of generalization would be to allow the firms to dispose of capacity after the demand is realized, as in the "Price Postponement with

\footnotetext{
${ }^{5}$ Radner (1992) provides an example of this in a simple model of Cournot competition under certain demand.

${ }^{6}$ Stuart (2004) provides an example.
}

Holdback" model of Van Mieghem and Dada (1999) and the "Holdback" model of Chod and Rudi (2003). In both these models, a market-clearing price is assumed to emerge once the firms have disposed of unwanted units. As before, a biform analysis can provide a foundation for such models provided that the core of the relevant game yields a market-clearing price. In the Van Mieghem and Dada model, this is the case. In the Chod and Rudi model, it is an open question.

\section{Conclusion}

In a biform analysis of competitive inventory decisions, a nonprocedural model of price competition, namely the core of the relevant cooperative game, yields three benefits. First, the source of two key newsvendor attributes, the overage/underage tension and the matching problem, can be clearly linked to the fixed-price assumption. Second, the unrestricted competition of the core can be used to evaluate models that assume market-clearing prices. For some models, the biform analysis, by utilizing the core, provides a foundation for the assumption. In other models, the analysis identifies that more justification is required. Finally, the biform analysis provides a purely gametheoretic Cournot analysis that can be extended to models with demand uncertainty. By contrast, Hviid (1991) showed that the pure-strategy, Cournot result of Kreps and Scheinkman (1983) does not extend when demand is uncertain.

\section{Acknowledgments}

The author thanks Adam Brandenburger, Awi Federgruen, Serguei Netessine, and Garrett van Ryzin for comments and discussions. Suggestions from an editor and two referees, all anonymous, are greatly appreciated. Financial support from Columbia Business School is gratefully acknowledged.

\section{Appendix}

Proof of LemMa 1.

Nonemptiness of the Core. We start by establishing that the core is nonempty. Consider the following allocation:

$$
\begin{gathered}
x_{i}=a_{i}[p-(k+c)], \quad i \in F, \\
x_{j}=w_{j}-p, \quad 1 \leq j \leq q, \quad \text { and } \\
x_{j}=0, \quad q<j \leq b,
\end{gathered}
$$

where $p \in\left[w_{q+1}, w_{q}\right]$ if $a_{N}<d_{N}, p \in\left[c, w_{q}\right]$ if $a_{N}=d_{N}$, and $p=c$ if $a_{N}>d_{N}$. 
First, recall that $R(N)=\min \left\{a_{N}, d_{N}\right\}=q$. Remembering that $p=c$ when $a_{N}>d_{N}$, we have, for any value of $a_{N}$,

$$
\sum_{i \in F} a_{i}[p-(k+c)]=-k a_{N}+\sum_{j=1}^{q}(p-c) .
$$

Thus,

$$
\begin{aligned}
x(N) & =\sum_{i \in F} a_{i}[p-(k+c)]+\sum_{j=1}^{q}\left(w_{j}-p\right) \\
& =-k a_{N}+\sum_{j=1}^{q}\left(w_{j}-c\right) \\
& =-k a_{N}+\sum_{j=1}^{R(N)} \chi_{N}(j)\left(w_{j}-c\right) \\
& =V(a)(N) .
\end{aligned}
$$

Next, note that for any $S \subset N$ such that $S \cap F=\varnothing$ or $S \cap T=\varnothing$, the allocation in (A1) trivially satisfies $x(S) \geq$ $V(a)(S)$. Therefore, consider an $S \subset N$ such that $S \cap F \neq \varnothing$ and $S \cap T \neq \varnothing$. If $R(S)>q$, note that $x_{j}=0$ for any $q<j \leq R(S)$. Thus,

$$
\begin{aligned}
x(S) & =\sum_{i \in S} a_{i}[p-(k+c)]+\sum_{j=1}^{q} \chi_{S}(j)\left(w_{j}-p\right) \\
& \geq-k a_{S}+(p-c) a_{S}+\sum_{j=1}^{R(S)} \chi_{S}(j)\left(w_{j}-p\right) \\
& \geq-k a_{S}+(p-c) \sum_{j=1}^{R(S)} \chi_{S}(j)+\sum_{j=1}^{R(S)} \chi_{S}(j)\left(w_{j}-p\right) \\
& =-k a_{S}+\sum_{j=1}^{R(S)} \chi_{S}(j)\left(w_{j}-c\right) \\
& =V(a)(S) .
\end{aligned}
$$

Thus, the allocation in (A1) is in the core, and the core is nonempty.

Uniform Price (Constant p), Buyers' Perspective. Consider each buyer $j$ such that $1 \leq j \leq q$. If a core allocation is consistent with the presence of a uniform price, it must be the case that each of these buyers receives value equal to $x_{j}=w_{j}-p$, namely, that $w_{j}-x_{j}$ is constant. The above nonemptiness proof showed that it was possible for a uniform price to emerge in the core of this game. We now show that a uniform price must emerge.

As a preliminary, we show that for any buyer $j$ such that $q<j \leq b, x_{j}=0$. Because the core is nonempty, no player can receive more than its marginal contribution. ${ }^{7}$ For any buyer $j>q, R(N \backslash\{j\})=R(N)$, so Equation (1) implies that $x_{j}=0$.

${ }^{7}$ Suppose to the contrary that $x_{i}>v(N)-v(N \backslash\{i\})$ in a core allocation. Because $x(N)=v(N)$ in the core, this would imply that $x(N \backslash\{i\})<v(N \backslash\{i\})$, contradicting another core condition.
Now consider two distinct buyers $j \leq q$ and $l \leq q$, and consider two firms with $a_{i}>0$, say $i$ and $i^{\prime}$. From Equation (1), we can construct distinct subsets $T_{1}, T_{2} \subset T$ such that $j \in T_{1}, l \in T_{2}$, and

$$
V(a)\left(\{i\} \cup T_{1}\right)+V(a)\left(\left\{i^{\prime}\right\} \cup T_{2}\right)+V(a)(S)=V(a)(N),
$$

where $S=N \backslash\left(\left\{i, i^{\prime}\right\} \cup T_{1} \cup T_{2}\right)$. Because the core is nonempty, it follows that

$$
\begin{gathered}
x\left(\{i\} \cup T_{1}\right)=V(a)\left(\{i\} \cup T_{1}\right), \\
x\left(\left\{i^{\prime}\right\} \cup T_{2}\right)=V(a)\left(\left\{i^{\prime}\right\} \cup T_{2}\right), \quad \text { and } \\
x(S)=V(a)(S) .
\end{gathered}
$$

Similarly, from Equation (1) again,

$$
\begin{gathered}
V(a)\left(\{i\} \cup\{l\} \cup T_{1} \backslash\{j\}\right)+V(a)\left(\left\{i^{\prime}\right\} \cup\{j\} \cup T_{2} \backslash\{l\}\right) \\
+V(a)(S)=V(a)(N)
\end{gathered}
$$

and

$$
x\left(\{i\} \cup\{l\} \cup T_{1} \backslash\{j\}\right)=V(a)\left(\{i\} \cup\{l\} \cup T_{1} \backslash\{j\}\right), \text { etc. }
$$

Combining equations,

$$
\begin{aligned}
& V(a)\left(\{i\} \cup\{l\} \cup T_{1} \backslash\{j\}\right)-V(a)\left(\{i\} \cup T_{1}\right) \\
& \quad=x\left(\{i\} \cup\{l\} \cup T_{1} \backslash\{j\}\right)-x\left(\{i\} \cup T_{1}\right) \\
& =x_{l}-x_{j} .
\end{aligned}
$$

From Equation (1),

$$
V(a)\left(\{i\} \cup\{l\} \cup T_{1} \backslash\{j\}\right)-V(a)\left(\{i\} \cup T_{1}\right)=w_{l}-w_{j},
$$

implying

$$
w_{l}-x_{l}=w_{j}-x_{j},
$$

and so there exists a $p$ equal to $w_{j}-x_{j}$ for $1 \leq j \leq q$.

Uniform Price, Firms' Perspective. Consider an arbitrary core allocation, and following Equation (A2), let $p=w_{j}-x_{j}$ for each buyer $j, 1 \leq j \leq q$. Let $F^{\prime}$ be the set of firms $i$ such that $a_{i}>0$, and for each $i \in F^{\prime}$, let

$$
p_{i}=\frac{x_{i}}{a_{i}}+(k+c) \text {. }
$$

From Equation (1), we can construct a partition $\left\{S_{i}\right\}_{i \in F^{\prime}}$ of $\{1, \ldots, q\}$ where $\left|S_{i}\right|=a_{i}$ if $d_{N} \geq a_{N}$ and $\left|S_{i}\right| \leq a_{i}$ if $d_{N}<a_{N}$, such that

$$
\sum_{i \in F^{\prime}} V(a)\left(\{i\} \cup S_{i}\right)=V(a)(N) .
$$

Then for each $i \in F^{\prime}$,

$$
x\left(\{i\} \cup S_{i}\right)=a_{i}\left(p_{i}-(k+c)\right)+\sum_{j \in S_{i}}\left(w_{j}-p\right) .
$$

Because the core is nonempty, and because $\sum_{i \in F^{\prime}} x\left(\{i\} \cup S_{i}\right)=$ $V(a)(N)$, for each $i \in F^{\prime}$,

$$
x\left(\{i\} \cup S_{i}\right)=\sum_{i \in F^{\prime}} V(a)\left(\{i\} \cup S_{i}\right),
$$


implying

$$
a_{i}\left(p_{i}-c\right)=\left|S_{i}\right|(p-c) .
$$

If $d_{N} \geq a_{N}$, then $\left|S_{i}\right|=a_{i}$, and $p_{i}=p$ all $i \in F^{\prime}$.

For the case $d_{N}<a_{N}$, i.e., $q<a_{N}$, summing Equation (A3) over all $i \in F^{\prime}$ yields

$$
\sum_{i \in F^{\prime}} a_{i} p_{i}-a_{N} c=q(p-c) .
$$

Solving Equation (A3) for $p_{i}$ and substituting into Equation (A4) yields

$$
\sum_{i \in F^{\prime}}\left|S_{i}\right| p-a_{N} c=q p-a_{N} c=q(p-c) .
$$

Since $q<a_{N}$, Equation (A5) implies that $p=c$, and Equation (A3) implies that $p_{i}=c=p$ for all $i \in F^{\prime}$.

The Bounds on $p$. If $q<a_{N}, p=c$ as just shown above. So, consider the case $a_{N} \leq d_{N}$ (implying $q=a_{N}$ ). Following Equation (A2), let $p=w_{j}-x_{j}$ for each buyer $j, 1 \leq j \leq q$. Then the core condition $x_{q} \geq 0$ implies that $p \leq w_{q}$. This upper bound is achievable because the allocations in (A1) are in the core.

For the lower bound, the marginal contribution of a buyer $l \leq q$ is

$$
\begin{aligned}
V(a) & (N)-V(a)(N \backslash\{l\}) \\
& =\sum_{j=1}^{R(N)} \chi_{N}(j)\left(w_{j}-c\right)-\sum_{j=1}^{R(N \backslash\{l\})} \chi_{N \backslash\{l\}}(j)\left(w_{j}-c\right) \\
& =\sum_{j=1}^{q} \chi_{N}(j)\left(w_{j}-c\right)-\sum_{j=1}^{q+1} \chi_{N \backslash\{l\}}(j)\left(w_{j}-c\right) \\
& =w_{l}-w_{q+1} \quad \text { if } a_{N}<d_{N}, \\
& =w_{l}-c \text { if } a_{N}=d_{N} .
\end{aligned}
$$

Because a player cannot receive more than its marginal contribution, namely $x_{l} \leq V(a)(N)-V(a)(N \backslash\{l\})$, and because $p=w_{l}-x_{l}$

$$
\begin{array}{ll}
p \geq w_{q+1} & \text { if } a_{N}<d_{N} \\
p \geq c & \text { if } a_{N}=d_{N} .
\end{array}
$$

These lower bounds are achievable because the allocations in (A1) are in the core.

Proof of Example 5. It suffices to show that $a_{i}$ is optimal for $\alpha_{i}=1$ and $\alpha_{i}=0$. This implies that there are four optimality conditions:

$$
\begin{aligned}
a_{i} w_{q} & \geq\left(a_{i}+1\right) w_{q+1} \\
a_{i} w_{q} & \geq\left(a_{i}-1\right) w_{q-1} \\
a_{i} w_{q+1} & \geq\left(a_{i}+1\right) w_{q+2} \\
a_{i} w_{q+1} & \geq\left(a_{i}+1\right) w_{q} .
\end{aligned}
$$

Because $w_{j}=K-m j$, these conditions can be written as:

$$
\begin{aligned}
\left(a_{i}+1\right) m & \geq w_{q}, \\
\left(a_{i}-1\right) m & \leq w_{q}, \\
\left(a_{i}+2\right) m & \geq w_{q}, \\
a_{i} m & \leq w_{q} .
\end{aligned}
$$

Consequently, we need to show only that

$$
\left(a_{i}+1\right) m \geq w_{q} \geq a_{i} m .
$$

Because $\operatorname{Kmod} m(f+1) \leq m$, there exists a positive integer $n$ such that $K=m n(f+1)+r, r \leq m$. For each $i \in F$, let $a_{i}=n$, and note that

$$
q=a_{N}=n f
$$

Thus,

$$
\begin{aligned}
w_{q} & =K-m q \\
& =m n(f+1)+r-m n f \\
& =m n+r .
\end{aligned}
$$

Because $a_{i}=n$, and because $r \leq m$, Equation (A6) then follows.

\section{References}

Anupindi, R., Y. Bassok, E. Zemel. 2001. A general framework for the study of decentralized distribution systems. Manufacturing Service Oper. Management 3 349-368.

Aumann, R. 1985. What is game theory trying to accomplish? K. J. Arrow, S. Honkapohja, eds. Frontiers of Economics. Basil Blackwell, Oxford, UK, 28-76.

Brandenburger, A., H. W. Stuart Jr. 1992. Modelling business strategy: A two-stage approach. H. W. Stuart Jr., ed. Nonequilibrium and non-procedural approaches to game theory. Ph.D. dissertation, Harvard University, Boston, MA.

Brandenburger, A., H. W. Stuart Jr. 1996. Value-based business strategy. J. Econom. Management Strategy 5 5-24.

Brandenburger, A., H. W. Stuart Jr. 2004. Biform games. Unpublished, New York University, New York.

Chod, J., N. Rudi. 2003. Resource flexibility with responsive pricing. Working paper, Simon School, University of Rochester, NY.

Davidson, C., R. Deneckere. 1986. Long-run competition in capacity, short-run competition in price, and the Cournot model. RAND J. Econom. 17 404-415.

Deneckere, R., J. Peck. 1995. Competition over price and service rate when demand is stochastic: A strategic analysis. RAND J. Econom. 26 148-162.

Edgeworth, F. 1881. Mathematical Psychics. Kegan Paul, London, UK.

Hviid, M. 1991. Capacity constrained duopolies, uncertain demand and non-existence of pure strategy equilibria. Eur. J. Political Econom. 7 183-190. 
Kaneko, M. 1976. On the core and competitive equilibria of a market with indivisible goods. Naval Res. Logist. Quart. 23 321-337.

Karjalainen, R. 1992. The newsboy game. Working paper, Wharton School, University of Pennsylvania, Philadelphia, PA.

Kreps, D., J. Scheinkman. 1983. Quantity pre-commitment and Bertrand competition yield Cournot outcomes. Bell J. Econom. 14 326-337.

Lippman, S. A., K. F. McCardle. 1997. The competitive newsboy. Oper. Res. 45 54-65.

Mahajan, S., G. van Ryzin. 2001. Inventory competition under dynamic consumer choice. Oper. Res. 49 646-657.

Moulin, H. 1995. Cooperative Microeconomics: A Game-Theoretic Introduction. Princeton University Press, Princeton, NJ.

Müller, A., M. Scarsini, M. Shaked. 2002. The newsvendor game has a non-empty core. Games Econom. Behavior 38 118-126.

Netessine, S., N. Rudi. 2003. Centralized and competitive inventory models with demand substitution. Oper. Res. Forthcoming.

Netessine, S., F. Zhang. 2003. Externalities through stocking decisions and supply chain efficiency. Working paper, Wharton School, University of Pennsylvania, Philadelphia, PA.

Parlar, M. 1988. Game-theoretic analysis of the substitutable product inventory problem with random demands. Naval Res. Logist. 35 397-409.
Radner, R. 1992. Transfer payments and the core of a profit-center game. P. Pasgupta, D. Gale, O. Hart, E. Maskin, eds. Essays in Honor of Frank Hahn. Cambridge University Press, Cambridge, UK, 316-339.

Shapley, L., M. Shubik. 1969. On market games. J. Econom. Theory 1 9-25.

Shapley, L., M. Shubik. 1972. The assignment game, I: The core. Internat. J. Game Theory 1 111-130.

Stuart Jr., H. W. 2004. Efficient spatial competition. Games Econom. Behavior 49 345-362.

Tirole, J. 1988. The Theory of Industrial Organization. MIT Press, Cambridge, UK.

Van Mieghem, J., M. Dada. 1999. Price versus production postponement: Capacity and competition. Management Sci. 45 1631-1649.

Vives, X. 1999. Oligopoly Pricing: Old Ideas and New Tools. MIT Press, Cambridge, MA.

von Neumann, J., O. Morgenstern. 1944. Theory of Games and Economic Behavior. Princeton University Press, Princeton, NJ.

Wang, Y., Y. Gerchak. 2001. Supply chain coordination when demand is shelf-space dependent. Manufacturing Service Oper. Management 3 82-87.

Wang, Q., M. Parlar. 1994. A three-person game theory model arising in stochastic inventory control theory. Eur. J. Oper. Res. 76 83-97. 